International Journal of Pure and Applied Mathematics

Volume 89 No. 3 2013, 401-408

ISSN: 1311-8080 (printed version); ISSN: 1314-3395 (on-line version)

url: http://www.ijpam.eu

doi: http://dx.doi.org/10.12732/ijpam.v89i3.11

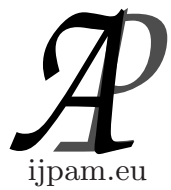

\title{
MACROSCOPIC WORMHOLES IN NONCOMMUTATIVE GEOMETRY
}

\author{
Peter K.F. Kuhfittig \\ Department of Mathematics \\ Milwaukee School of Engineering \\ Milwaukee, Wisconsin, 53202-3109, USA
}

\begin{abstract}
The purpose of this paper is to show that wormholes in noncommutative geometry can be macroscopic, based in part on an earlier study. The necessary violation of the weak energy condition is attributable to the noncommutative-geometry background rather than to the use of "exotic matter." The zero tidal forces make the wormhole suitable for humanoid travelers. Also discussed is the stability to linearized radial perturbations.
\end{abstract}

AMS Subject Classification: $83 \mathrm{C} 05$

Key Words: traversable wormholes, noncommutative geometry

\section{Introduction}

An important outcome of string theory is the realization that coordinates may become noncommutative operators on a $D$-brane $[12,9]$. The result is a fundamental discretization of spacetime due to the commutator $\left[\mathbf{x}^{\mu}, \mathbf{x}^{\nu}\right]=i \theta^{\mu \nu}$, where $\theta^{\mu \nu}$ is an antisymmetric matrix, similar to the way that the Planck constant $\hbar$ discretizes phase space. Noncommutativity replaces point-like objects by smeared objects $[10,5,11]$. The goal is to eliminate the divergences that 
normally appear in general relativity. The smearing effect can be accomplished by using a Gaussian distribution of minimal length $\sqrt{\theta}$ instead of the Dirac delta function in the following manner: the energy-density of the static and spherically symmetric and particle-like gravitational source has the form $[5,8]$

$$
\rho(r)=\frac{M}{(4 \pi \theta)^{3 / 2}} e^{-r^{2} / 4 \theta} .
$$

Here the mass $M$ is diffused throughout the region of linear dimension $\sqrt{\theta}$ due to the uncertainty. The noncommutative geometry is an intrinsic property of spacetime and does not depend on particular features such as curvature.

Noncommutative geometry has proved to be an effective tool in several areas. Thus Garattini and Lobo [1] obtained a self-sustained wormhole using a semiclassical analysis. Kuhfittig [3] showed that a special class of thin-shell wormholes posses small regions of stability around the thin shell even though they are unstable in classical general relativity. Rahaman et al. [6] concluded that a noncommutative-geometry background is sufficient for producing stable circular orbits in a typical galaxy without the need for dark matter.

Turning our attention now to traversable wormholes, recall that the line element describing a Morris-Thorne wormhole is given by [4]

$$
d s^{2}=-e^{\Phi(r)} d t^{2}+\frac{d r^{2}}{1-b(r) / r}+r^{2}\left(d \theta^{2}+\sin ^{2} \theta d \phi^{2}\right) .
$$

Here $b=b(r)$ is called the shape function and $\Phi=\Phi(r)$ the redshift function, which must be everywhere finite to prevent an event horizon. For the shape function we must have $b\left(r_{0}\right)=r_{0}$, where $r=r_{0}$ is the radius of the throat of the wormhole. In addition, $b^{\prime}\left(r_{0}\right)<1$ and $b(r)<r$ to satisfy the flare-out condition [4]. The flare-out condition, in turn, can only be satisfied by violating the weak energy condition, to be discussed below. For ordinary matter, this violation can only be accomplished by the use of "exotic matter."

The parameter $\theta$ occurring in noncommutative geometry suggests the existence of a very small scale leading to wormholes that are actually microscopic. This would require a semi-classical framework, as carried out in detail in reference [1]. Using $r / \sqrt{\theta}$ instead of $r$ for the horizontal axis in references $[5,11,1]$ also suggests that small values of $r$ are intended. Using reference [7] as a starting point, it is shown in this paper that such a wormhole can be macroscopic and that the noncommutative-geometry background replaces the exotic matter. Moreover, the model presented has zero tidal forces, thereby making it suitable for humanoid travelers. Finally, it is proposed that these wormholes can be made stable to linearized radial perturbations. 


\section{The Solutions}

To make the wormholes suitable for humanoid travelers, it is desirable to have a constant redshift function, so that $\Phi^{\prime} \equiv 0$, the so-called zero-tidal-force solution proposed in reference [4]. Given this condition, the Einstein field equations become

$$
\begin{aligned}
\rho(r) & =\frac{1}{8 \pi} \frac{b^{\prime}(r)}{r^{2}}, \\
p_{r}(r) & =-\frac{1}{8 \pi} \frac{b(r)}{r^{3}},
\end{aligned}
$$

and

$$
p_{t}(r)=\frac{1}{8 \pi}\left(1-\frac{b(r)}{r}\right) \frac{b(r)-r b^{\prime}(r)}{2 r^{2}[r-b(r)]} .
$$

Here $\rho(r)$ is the energy-density, equation $(1), p_{r}(r)$ is the radial pressure, and $p_{t}(r)$ the lateral pressure.

Making use of equation (1), we obtain the shape function

$$
b(r)=\frac{M}{\sqrt{\pi}}\left[2 \sqrt{\pi} \operatorname{erf}\left(\frac{r}{2 \sqrt{\theta}}\right)-\frac{2 r}{\sqrt{\theta}} e^{-r^{2} / 4 \theta}+C\right],
$$

where $C$ is an integration constant. (How $M$ is related to the mass of the wormhole will be seen at the end of Section 3.) This solution is also given in reference [7], where it is shown that the wormhole spacetime is asymptotically flat.

Since $C$ is an integration constant, mathematically $b(r)$ in equation (6) is a valid solution for every $C$. The flare-out condition, among others, is a physical requirement that is satisfied only for a certain range of values of $C$. To show that the wormhole is macroscopic, we use a graphical approach, as in reference [7], by assigning values to the parameters that are not only typical but help produce useful and revealing graphs. Since we are using geometrized units (with $c=G=1$ ), the mass $M$ takes on units of length; so it is desirable to measure distances in meters, as in references [4]. Moreover, unlike references [5, 11, 1], we plot $b(r)$ against $r$, rather than $r / \sqrt{\theta}$. As an illustration, suppose we choose $M=0.05, \theta=0.001$, and $C=3$ to obtain $b(r)$ in Figure 1 .

The throat of the wormhole is located at $r=r_{0}$, where $B(r)=b(r)-r$ intersects the $r$-axis, shown in Figure 2 . The throat radius turns out to be $r_{0}=$ $0.18462 \mathrm{~m}$, which is definitely macroscopic, a conclusion already deducible from reference [7]. (The value can be readily increased by adjusting the parameters.) According to Figure 2, for $r>r_{0}, B(r)<0$, so that $b(r)-r<0$, which implies 


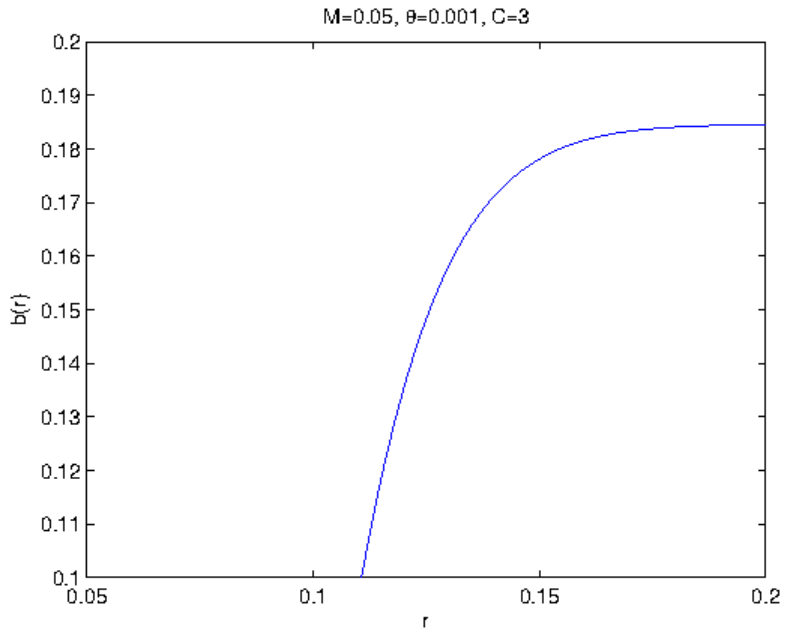

Figure 1: The shape function of the wormhole

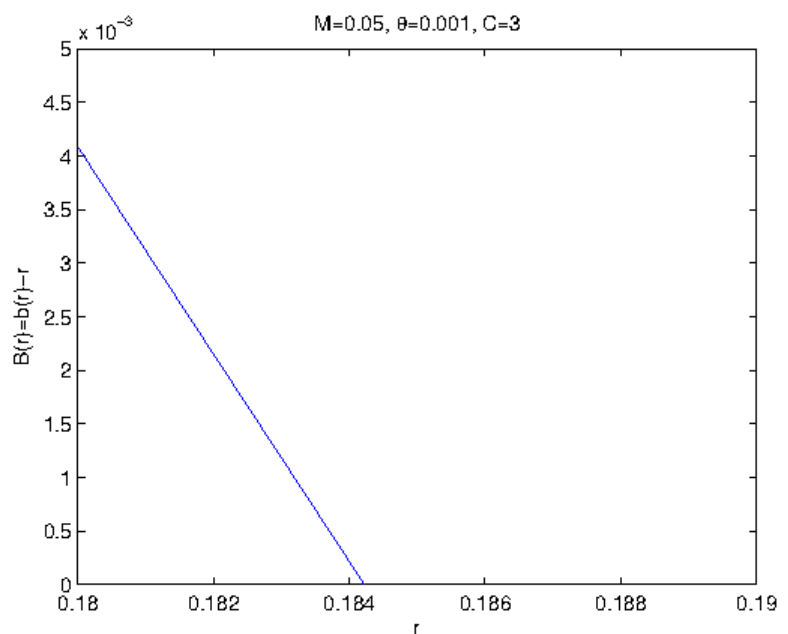

Figure 2: The throat radius is the $r$-intercept of $B(r)=b(r)-r$ 
that $b(r) / r<1$ for $r>r_{0}$. Also, since $B(r)$ is decreasing, we have $B^{\prime}\left(r_{0}\right)<0$ and hence $b^{\prime}\left(r_{0}\right)<1$. The flare-out condition is therefore satisfied.

\section{Violation of the Weak Energy Condition}

Next, recall from reference [4] that the condition $b^{\prime}\left(r_{0}\right)<1$ implies that $\rho+p_{r}<$ 0 , a condition that is normally associated with exotic matter. However, there exist other possibilities. For example, phantom energy is characterized by the equation of state $p=w \rho, w<-1$, which yields $\rho+p=\rho(w+1)<0$. In the present situation, equations (4) and (6) imply that

$$
p_{r}(r)=-\frac{1}{8 \pi r^{3}} \frac{M}{\sqrt{\pi}}\left[2 \sqrt{\pi} \operatorname{erf}\left(\frac{r}{2 \sqrt{\theta}}\right)-\frac{2 r}{\sqrt{\theta}} e^{-r^{2} / 4 \theta}+C\right] .
$$

In reference [7], it is shown graphically that $\rho+p_{r}<0$ near the throat, i.e.,

$$
\begin{aligned}
\rho+p_{r}=\frac{M}{(4 \pi \theta)^{3 / 2}} & e^{-r^{2} / 4 \theta} \\
& -\frac{1}{8 \pi r^{3}} \frac{M}{\sqrt{\pi}}\left[2 \sqrt{\pi} \operatorname{erf}\left(\frac{r}{2 \sqrt{\theta}}\right)-\frac{2 r}{\sqrt{\theta}} e^{-r^{2} / 4 \theta}+C\right]<0
\end{aligned}
$$

for certain choices of the parameters. For the values in the above example $(M=0.05, \theta=0.001$, and $C=3)$, we obtain, at the throat $r_{0}=0.18462$, $\rho+p_{r}=-0.657$. It is important to realize that this is a direct consequence of the noncommutative-geometry background, rather than to some form of "exotic matter." The reason is that we are not following the strategy in reference [4], tailoring $b(r)$ and $\Phi(r)$ to produce a traversable wormhole, while letting $\rho, p_{r}$, and $p_{t}$ dangle. Instead, $b(r)$ and $p_{r}(r)$ were obtained from $\rho(r)$ in equation (1) and the Einstein field equations; $p_{t}(r)$ is derived in reference [7].

Since the smearing effect is heavily influenced by the size of $\theta$, smaller values should be considered. (Recall that $\theta=0.001$ was chosen mainly for producing useful graphs.) It turns out, however, that reducing $\theta$ seems to have little effect on the outcome with one notable exception: as $\theta$ gets smaller, $b^{\prime}\left(r_{0}\right)$ gets closer to zero. This is to be expected since $\lim _{\theta \rightarrow 0} b(r)=2 M+M C / \sqrt{\pi}$, a constant. The slow flaring out, however, could have an effect on the proper distance around the throat. To provide a numerical check on our example, consider the coordinate distance given by the interval $[0.18462 \mathrm{~m}, 100 \mathrm{~m}]$. The corresponding proper distance is

$$
\int_{0.18462}^{100} \frac{d r}{\sqrt{1-b(r) / r}} \approx 100.61 \mathrm{~m}
$$


So the proper distance is not much larger than the coordinate distance.

Remark. The above limit, $\lim _{\theta \rightarrow 0} b(r)=2 M+M C / \sqrt{\pi}=\lim _{r / \sqrt{\theta} \rightarrow \infty} b(r)=$ $2 M+M C / \sqrt{\pi}$, shows that $2 M+M C / \sqrt{\pi}=2 M_{S}$, the Schwarzschild limit. So in the above example, since $M=0.05$ and $C=3$, we obtain $M_{S}=0.092 \mathrm{~m}$, which may be viewed as the mass of the wormhole relative to a distant observer.

\section{Stability}

It is pointed out in reference [2] that it is possible in principle to construct a wormhole that is stable to linearized radial perturbations. The wormhole has to meet the usual conditions at the throat, but when joined to an external Schwarzschild spacetime at the junction surface $r=a>r_{0}$, some additional conditions need to be met: to be a stable wormhole, the redshift function $\Phi=\Phi(r)$ must satisfy the conditions

$$
\Phi(a-)=\Phi(a+) \quad \text { and } \quad \Phi^{\prime}(a-)=\frac{M}{a(a-2 M)},
$$

referring to the left- and right-hand limits, respectively. [In other words, $\Phi_{\text {internal }}(a)=\Phi_{\text {external }}(a)$ and $\Phi_{\text {internal }}^{\prime}(a)=\Phi_{\text {external }}^{\prime}(a)$.] It is also shown in reference [2] that the shape function $b=b(r)$ must be an increasing function of $r$ having a continuous second derivative and that it must attain a maximum value at $r=a$. For such a shape function, the stability criterion is surprisingly simple:

$$
b^{\prime \prime}(a)<-\frac{8 M}{a^{2}} .
$$

The implication is that from the standpoint of theoretical construction, this condition would not be difficult to meet. Joining our earlier solution, namely $\Phi \equiv$ constant (i.e., $\Phi^{\prime} \equiv 0$ ) and equation (6), to this new solution will require transitional curves that smoothly connect, respectively, the redshift and shape functions. The result is a stable wormhole.

This construction does not depend on any particular properties of the wormhole, suggesting that any wormhole can be made stable to linearized radial perturbations.

\section{Conclusion}

It is shown in this paper that it is possible in principle to design or construct a traversable wormhole with zero tidal forces in a noncommutative-geometry 
setting. Not only is the wormhole spacetime macroscopic and asymptotically flat, the violation of the weak energy condition is due to the noncommutative geometry, rather than to "exotic matter." So if string theory is correct, then the laws of physics seem to allow macroscopic traversable wormholes with zero tidal forces.

It is also proposed in this paper that any wormhole of the Morris-Thorne type can be modified to become stable to linearized radial perturbations.

\section{References}

[1] R. Garattini, F.S.N. Lobo, Self-sustained traversable wormholes in noncommutative geometry, Phys. Lett. B, 671, No. 1 (2009), 146-152, doi: 10.1016/j.physletb.2008.11.064.

[2] P.K.F. Kuhfittig, Theoretical construction of stable traversable wormholes, Central Eur. J. Phys., 8, No. 3 (2010), 364-368, doi: 10.2478/s11534-0090099-4.

[3] P.K.F. Kuhfittig, On the stability of thin-shell wormholes in noncommutative geometry, Advances in High Energy Physics, 2012 (2012), 462493, 12 pages, doi: 10.1155/2012/462493.

[4] M.S. Morris, K.S. Thorne, Wormholes in spacetime and their use for interstellar travel: A tool for teaching general relativity, Am. J. Phys., 56 (1988), 395-412, doi: dx.doi.org/10.1119/1.15620.

[5] P. Nicolini, A Smailagic, E. Spalluci, Noncommutative geometry inspired Schwarzschild black hole, Phys. Lett. B, 632, No. 4 (2006), 547-551, doi: 10.1016/j.physletb.2005.11.004.

[6] F. Rahaman, P.K.F. Kuhfittig, K. Chakraborty, A.A. Usmani, S. Ray, Galactic rotation curves inspired by a noncommutative-geometry background, Gen. Rel. Grav., 44, No. 4 (2012), 905-916, doi: 10.1007/s10714011-1320-5.

[7] F. Rahaman, P.K.F. Kuhfittig, S. Ray, S. Islam, Searching for higherdimensional wormholes with noncommutative geometry, Phys. Rev. D, 86, No. 10 (2012), 106010, 7 pages, doi: 10.1103/PhysRevD.86.106010.

[8] M. Rinaldi, A new approach to non-commutative inflation, Class. Quantum Grav., 28, number 10 (2011), 105022, 10 pages, doi: 10.1088/0264$9381 / 28 / 10 / 105022$. 
[9] N. Seiberg, E. Witten, String theory and noncommutative geometry, JHEP, 9909 (1999), 032, doi: 10.1088/1126-6708/1999/09/032.

[10] A. Smailagic, E. Spalluci, Feynman path integral on the non-commutative plane, J. Phys. A, 36, number 33 (2003), L467-L471, doi: 10.1088/0305$4470 / 36 / 33 / 101$.

[11] E Spallucci, A. Smailagic, P. Nicolini, Non-commutive geometry inspired higher-dimensional charged black holes, Phys. Lett. B, 670, No. 4-5 (2009), 449-454, doi: 10.1016/j.physletb.2008.11.030.

[12] E. Witten, Bound states of strings and p-branes, Nucl. Phys. B, 460, No. 2 (1996), 335-350, doi: 10.1016/0550-3213(95)00610-9. 